\title{
Firing Patterns and Synaptic Potentials of Identified Giant Aspiny Interneurons in the Rat Neostriatum
}

\author{
C. J. Wilson, H. T. Chang, and S. T. Kitai \\ Department of Anatomy and Neurobiology, University of Tennessee, Memphis College of Medicine, Memphis, Tennessee \\ 38163
}

Intracellular recordings were made in vivo from 9 giant aspiny neurons in the neostriatum of urethane-anesthetized rats. The cells were identified by intracellular staining with HRP or biocytin. The neurons exhibited morphological features typical of neostriatal cholinergic interneurons. Six of the cells were obtained from intact animals, while 3 were recorded from rats with ipsilateral hemidecortications.

Giant aspiny neurons were characterized by their slow irregular but tonic (3-10/sec) spontaneous activity and longduration action potentials. Examination of the underlying membrane potential trajectories during spontaneous firing revealed that individual action potentials were triggered from spontaneous small (1-5 mV) depolarizing potentials. These spontaneous potentials exhibited the voltage sensitivity of ordinary EPSPs. They were much less frequent during the 80-200 msec pause in tonic afferent input that follows the excitation evoked by cortical or thalamic stimulation, and were decreased in frequency in decorticate animals. Their rise times and half-widths matched those expected for unitary synaptic potentials placed proximally on the surface of the neurons. Low-intensity stimulation of neostriatal afferents produced small short-latency EPSPs that appeared to be composed of responses identical to the spontaneous depolarizing potentials. The latencies of the EPSPs evoked from the cerebral cortex and thalamus were consistent with a monosynaptic input from both structures, but the maximal size of the EPSPs was much smaller than that evoked in spiny neurons, suggesting that a smaller number of afferent inputs make synapses with each of the aspiny cells.

Giant aspiny neurons exhibited much larger input resistances and longer time constants than spiny neostriatal neurons. They also exhibited relatively linear steady-state current-voltage relationship compared to spiny projection cells. Input resistances ranged from 71-105 $\mathrm{M} \Omega$, and time constants ranged from 17.8-28.5 msec. Analysis of the charging transients in response to current pulses yielded estimates of dendritic length of approximately 1 length constant. Repetitive firing of the neurons was limited by a powerful spike afterhyperpolarization and by a strong spike frequency adaptation.

The sensitivity of the giant aspiny interneuron to a rela-

Received June 7, 1989; revised July 27, 1989; accepted July 28, 1989

This work was supported by NIH grant NS20473 and RCDANSO 1078 to C.J.W., AG05944 to H.T.C., and NS20702 to S.T.K.

Correspondence should be addressed to Charles J. Wilson, Department of Anatomy and Neurobiology, University of Tennessee, Memphis, 875 Monroe Avenue, Memphis, TN 38163.

Copyright (c) 1990 Society for Neuroscience $0270-6474 / 90 / 100508-12 \$ 02.00 / 0$ tively small number of proximal afferent synaptic contacts, its tonic firing, and its widespread dendritic and axonal fields place it in an excellent position to act as a modulator of the excitability of neostriatal projection neurons in advance of the onset of movement-related neostriatal activity.

Between 6 and 9 cell categories have been constructed for the neurons of the mammalian neostriatum based on their somatodendritic morphology in sections stained with the Golgi method (e.g., Kemp and Powell, 1971; DiFiglia et al., 1976; Chang et al., 1982). In these studies, the main group of projection neurons, the spiny cells, are viewed as a single category of cells, and the remaining cells are all aspiny neurons. Most of the aspiny cells are believed to be interneurons, and the differences in the numbers of cell classes described by the various authors are primarily in their schemes for dividing up the interneurons. This has been a difficult task because, despite their morphological and cytochemical diversity, the aspiny neurons are relatively rare. They account for as little as $5 \%$ of the total cell population in rats and cats (e.g., Kemp and Powell, 1971; Graveland and DiFiglia, 1985).

An enormous sampling bias against the projection cells would be required to obtain a reasonable sample of aspiny cells by random sampling. Despite their small size, the neostriatal spiny neurons are relatively resistant to damage by microelectrodes, so there is no sampling bias against them that would facilitate recording from aspiny neurons. In addition, there have been no neurophysiological criteria to allow identification of aspiny neurons during intracellular recordings experiments. Elimination of spiny projection neurons after identifying them by antidromic activation has not been reliable. For reasons that are not yet clear, many spiny projection neurons identified by intracellular injection of HRP, and even by following their axons from the neostriatum, do not respond antidromically to stimulation of substantia nigra or globus pallidus (e.g., Liles, 1974; Fuller et al., 1975; Preston et al., 1980; Chang et al., 1981). It is therefore not surprising that there have been no intracellular recording studies of the properties of identified neostriatal interneurons.

Nevertheless, the physiological properties of aspiny neurons are of some functional importance. For example, among the various types of aspiny neurons is the cholinergic neuron, whose activity has long been postulated as the focus of synaptic interactions responsible for the therapeutic effect of a variety of treatments in Parkinson's disease, Huntington's disease, tardive dyskinesia, and other disorders (e.g., see review by Groves, 1983). Recognition of the importance of aspiny cells and of their variety in the neostriatum is rapidly increasing as new cytochemical markers reveal categories of cells that stain specifically for var- 
ious other neuroactive substances known to be important in brain function.

Extracellular recordings have the advantage of allowing the collection of very large samples, in which even rare cell types may be detected. Most neurons in the neostriatum have very low firing rates and may be silent for long periods of time. Their silence is interrupted by episodal firing that may be associated with performance of a learned task (e.g., DeLong, 1973). This firing pattern has previously been shown to be typical of the spiny projection neurons (Wilson and Groves, 1981). A minority of neurons show a tonic firing pattern, characterized by average firing rates as high as $10 \mathrm{~Hz}$, with increases and decreases in rate that may be temporally related to the presentation of a sensory cue used as a signal for the animals to perform a learned task (Kimura et al., 1984). The identity of the ncurons firing in this pattern and their position within the circuitry of the neostriatum are unknown, but they have been presumed to be aspiny neurons.

Over the past 8 years of intracellular recording and staining of neostriatal neurons, we have collected a sample of large aspiny neurons similar enough in their morphological and physiological properties to be considered representative of a single cell type. These cells appear to correspond to the tonically active neurons described by Kimura et al. (1984). These labeled neurons also show morphological features identical to those of the large choline acetyltransferase-containing neurons described in immunocytochemical studies (Bolam et al., 1984; Phelps et al., 1985; DiFiglia, 1987). In this report we describe the spontaneous firing, synaptic responses to afferent stimulation, and some of the membrane properties of these cells. A detailed description of the morphological features of the cells will be reported elsewhere.

\section{Materials and Methods}

The cells were impaled in the course of intracellular recording experiments designed for other purposes. These cells were found only very occasionally ( 1 per year on average), and so no experiment was specifically designed for their analysis. Consequently, they were obtained under a variety of conditions. In all cases, the cells were recorded in male rats, weighing between 250 and $550 \mathrm{gm}$ and anesthetized with Urethane $(1.3 \mathrm{gm} / \mathrm{kg})$ supplemented with $\mathrm{ketamine}(35 \mathrm{mg} / \mathrm{kg}$, i.m., every $1 \mathrm{hr})$ or a combination of ketamine and xylazine $(35 \mathrm{mg} / \mathrm{kg} \mathrm{ke}-$ tamine, $6 \mathrm{mg} / \mathrm{kg}$ xylazine, i.m., every $1 \mathrm{hr}$ ). The animals were fixed in a stereotaxic device, and stimulating electrodes were implanted and attached to the skull with dental cement. The cerebrospinal fluid was drained, and the animal was suspended by a clamp placed on the base of the tail. Body temperature was maintained at $36.5 \pm 1{ }^{\circ} \mathrm{C}$. In 2 of the 9 animals, the ipsilateral cerebral cortex had been removed acutely (between 2 and $6 \mathrm{hr}$ earlier) by aspiration. A similar cortical lesion has been made $3 \mathrm{~d}$ preceding the experiment in another animal. The cortical lesions were very large, including the entire rostral pole of the cortex, and the frontal and parietal cortices lateral to within $1 \mathrm{~mm}$ of the rhinal sulcus and medial to within $0.5 \mathrm{~mm}$ of the midline. The corpus callosum was anatomically intact in all 3 cases, and the presence of synaptic responses evoked by stimulation of contralateral cerebral cortex stimulation was verified in 2 of these.

Stimulating electrodes were placed in the cerebral peduncle at the level of the substantia nigra or in the substantia nigra itself (6 rats), the ipsilateral cerebral cortex (4 rats), the ipsilateral parafascicular thalamic nucleus (6 rats), or the contralateral cerebral cortex ( 2 rats). Stimulating electrodes were pairs of insect pins (00 gauge) insulated except for 0.25 $\mathrm{mm}$ at the tips with epoxylite. Stimuli were single bipolar $0.1 \mathrm{msec}$ duration current pulses, $0.01-1.0 \mathrm{~mA}$ in amplitude.

Intracellular recordings were obtained using glass micropipettes broken under microscopic control to have tip diameters less than $0.5 \mu \mathrm{m}$. They were filled with 4\% HRP (Sigma Type VI) in $0.5 \mathrm{~m}$ potassium methylsulfate and $0.05 \mathrm{M}$ Tris buffer $(\mathrm{pH} 7.6)$ or with $2 \%$ biocytin in 1 M potassium acetate (Horikawa and Armstrong, 1988). They had re- sistances between 30 and $80 \mathrm{M} \Omega$. Cells were labeled by passage of positive current pulses, $1.0-3.0 \mathrm{nA}$ in amplitude and $150 \mathrm{msec}$ in duration, at a rate of $3 / \mathrm{sec}$ for $2-20 \mathrm{~min}$.

After completion of the recording experiment, the brains were fixed by intracardial perfusion of an aldehyde fixative and stored in fixative overnight. Sections through the region of the injected neurons were cut at a thickness of $50 \mu \mathrm{m}$ on a Vibratome and collected into phosphate buffer or buffered saline. Sections containing cells injected with biocytin were treated in a solution of avidin-biotin-HRP complex (ABC, 1:100 dilution, Vector) and $0.25 \%$ Triton-X100 in PBS for $4 \mathrm{hr}$. All sections were reacted with $\mathrm{DAB}$ (3'-diaminobenzidine, $0.05 \%$, and hydrogen peroxide, $0.03 \%$ ) for $5-25 \mathrm{~min}$. Sections containing injected neurons were identified by microscopic observation in buffer and were treated with osmium tetroxide $(0.1-1 \%$ depending upon whether they were to be prepared for electron microscopy), dehydrated, and embedded in plastic between glass microscope slides and coverslips. Neurons were drawn at $600 \times$ using a $60 \times$ oil-immersion objective (n.a. 1.4) and a drawing tube.

\section{Results}

\section{Identification of neurons}

Nine cells were identified as giant aspiny neurons on the basis of their morphological features after intracellular staining with HRP ( 7 cells) or biocytin ( 2 cells). These represent about $2.6 \%$ of the sample of approximately 350 neostriatal neurons we stained intracellularly over the same time period. On the basis of observations on Nissl-stained sections from the same animals and from the previous work of others (e.g., Kemp and Powell, 1971), cells large enough to belong to the same cell class as these neurons account for only $1-2 \%$ of the total neuron population in the rostral head of the rat caudate putamen, where the recordings were made. Thus, there is no sampling bias against these cells, and in fact, cells of this type are probably slightly overrepresented in intracellular recording experiments, perhaps due to their large size. The possibility of a sampling bias in their favor is also suggested from the quality of the recordings. In general, intracellular recordings from giant aspiny neurons were of higher quality and were maintained for longer periods than recordings from medium spiny neurons, which made up the overwhelming majority of intracellularly stained neostriatal neurons. All of the neurons whose responses are reported here had membrane potentials in excess of $50 \mathrm{mV}$ and action potentials in excess of $55 \mathrm{mV}$ when firing spontaneously. All of the neurons had action potential amplitudes in excess of their membrane potentials (spike overshoot). Four of them were held for over $2 \mathrm{hr}$ with no sign of deterioration.

The morphological features of a representative giant aspiny neuron as seen after intracellular staining are shown in Figure 1. The somata were elongated and generally aligned in the rostrocaudal dircetion. Measurements of somatic sizc are difficult because the cell bodies cannot easily be differentiated from the 2-4 large primary dendrites, but the cells ranged in cross-sectional area from 445 to $586 \mu \mathrm{m}^{2}$ (mean, $506 \mu \mathrm{m}^{2}$ ). For comparison, a sample of 61 neurons was collected from sections of rat neostriatum stained using immunocytochemistry for choline acetyl transferase (ChAT), cut in the same plane, treated similarly as the intracellularly stained neurons, and measured in the same way. The cells in this sample ranged from 308 to $700 \mu \mathrm{m}^{2}$ in cross section, with a mean of $446 \mu \mathrm{m}^{2}$ and a standard deviation of 86.2. Thus, the injected neurons in this sample are approximately the same size as the cholinergic interneurons and are thus among the largest neurons in the rat neostriatum. The intrasomatic placement of the recording electrode in the neuron shown in Figure 1 is indicated by the peroxidase-positive erythrocytes that mark the track of the electrode. 
Figure 1. A photomicrograph of an intracellularly stained giant aspiny neuron in a coronal section from the rat neostriatum. The section is stained with osmium tetroxide, which accounts for the staining of the capsular fiber bundles. The site of recording in this neuron is apparent due to the numerous red blood cells, stained darkly because of their peroxidase activity, along the electrode track. Only a small part of the dendritic field is visible in this micrograph.

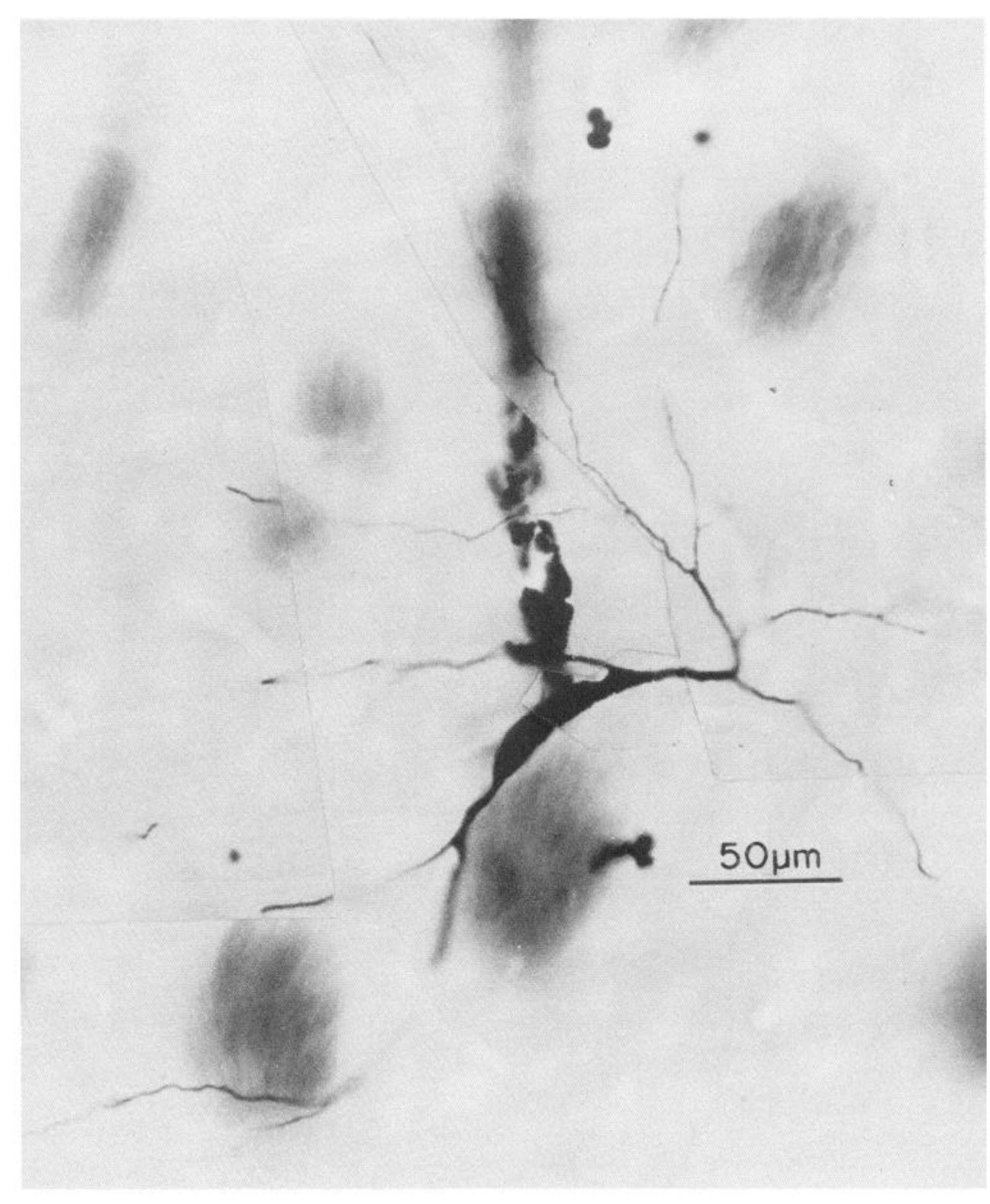

The large dendritic trunks branched repeatedly to fill a space extending approximately $500 \mu \mathrm{m}$ in the dorsoventral and mediolateral directions, and 750-1000 $\mu \mathrm{m}$ in the rostrocaudal directions. This arrangement of dendrites is shown in coronal section in the drawing in Figure 2. This dendritic field orientation in the rostrocaudal direction is probably partly the result of the locations of our neurons. All the recordings were made in the rostral head of the caudate-putamen, where afferent and efferent fibers are all aligned rostrocaudally. Thus, the dendritic trees of the aspiny neurons may conform to the general orientation of the tissue in this region of the neostriatum. The distal dendrites of the aspiny neurons exhibited numerous fine, short side branches, varicosities, and spine-like appendages. Near their terminals, many dendrites branched repeatedly to form a complex terminal thicket, similar to those reported for neurons in the substantia nigra (Grofova et al., 1982). The axons of the aspiny cells in this sample all arose from a primary dendrite at least $20 \mu \mathrm{m}$ from the soma. In the early part of their trajectories, the axons appeared identical to the dendrites from which they arose. It was only from observation of the more distal branching pattern that their axonal identity could be ascertained. They coursed farther between branch points than did the dendrites and gave rise to equal-sized daughter branches. The axonal arborizations of the neurons were very dense and extended over a large region of the neostriatum, usually even larger than the volume occupied by the dendritic field.

In intracellular recordings, giant aspiny neurons could be distinguished from the more commonly encountered spiny projection neurons in 2 ways. One was their tonic spontaneous firing, which was especially evident in intact animals. The other was the long duration and characteristic shape of their action potentials.

\section{Spontaneous firing pattern}

In contrast to the more common spiny neurons, which were generally silent or exhibited phasic activity, all of the giant aspiny neurons recorded in intact animals fired tonically in the absence of stimulation. The firing of these cells was not periodic or 


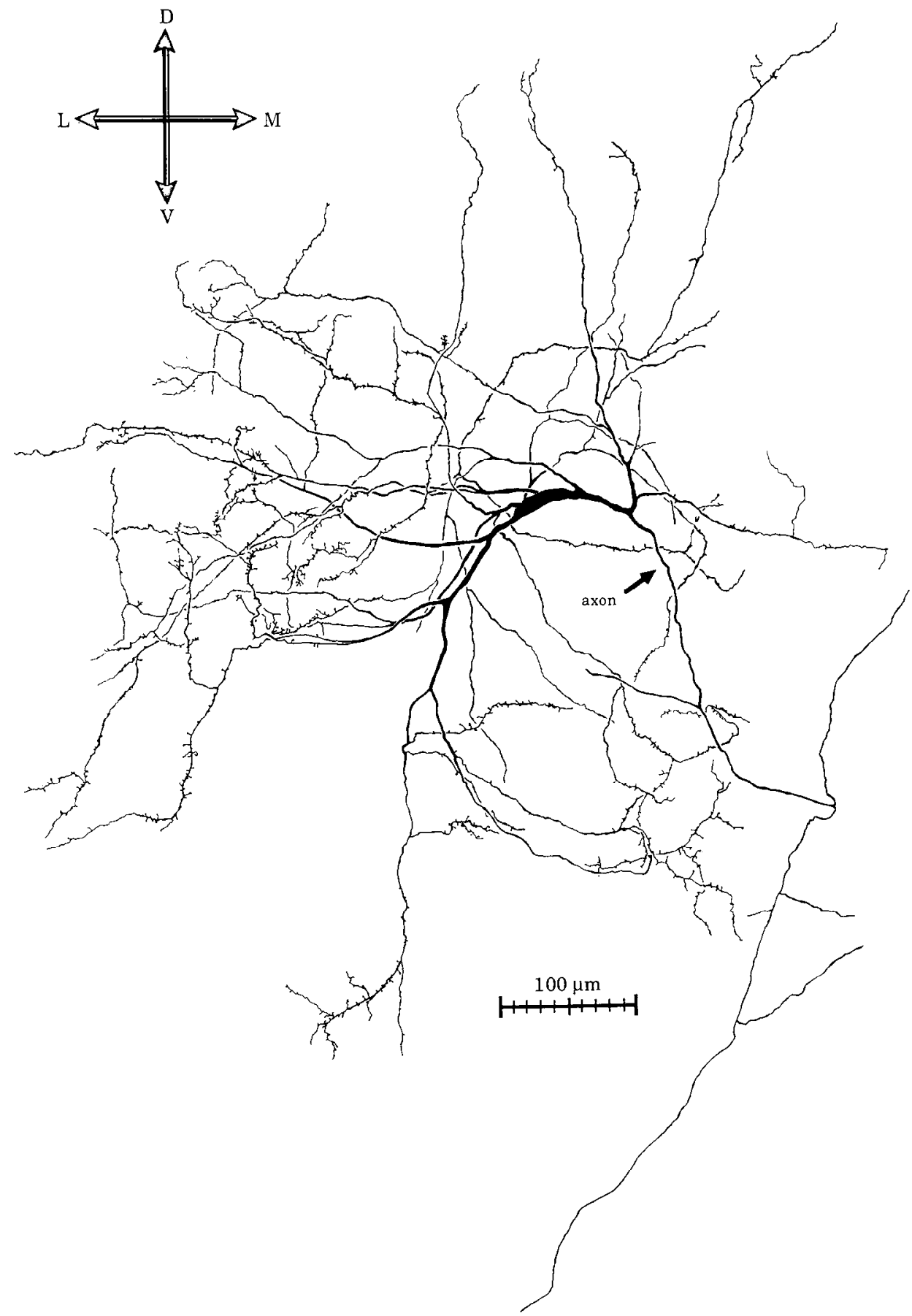

Figure 2. Reconstruction of the entire dendritic tree of the cell shown in Figure 1 . The extent of the dendritic field in the mediolateral and dorsoventral directions is apparent. Nineteen sections, each $50 \mu \mathrm{m}$ thick, were required for reconstruction of the dendritic field in the rostrocaudal direction. The axon of this cell had a series of successive bifurcations, with resulting branches of approximately equal size. All branches of the axon became extremely fine after only a few such bifurcations. Only the first few of the largest axonal branches are shown. bursty, but was a maintained irregular firing at rates of 3-10/ $\mathrm{sec}$. This firing, as seen in intracellular recordings, is illustrated in Figure $3 A$. Spontaneous action potentials were seen to arise from small spontaneous depolarizing potentials, with amplitudes of $0.5-5.0 \mathrm{mV}$. These depolarizing potentials likewise were irregular in occurrence, and most were subthreshold for generation of action potentials. Larger spontaneous potentials often arose by summation of the smaller ones, as indicated by an inflection in the rising phase of the depolarization, and a prolongation of its time course. As the membrane potential of the giant cells was never more than $5 \mathrm{mV}$ from the action potential threshold, and was more commonly only 1 or $2 \mathrm{mV}$ below the value required to trigger a spike, the spontaneous depolarizing potentials often could trigger action potentials. This too is in contrast with the more common spiny projection neurons, whose membranes are commonly more than $10 \mathrm{mV}$ from threshold in the same preparation. Hyperpolarization of the membrane of the giant aspiny ncurons by only a few millivolts completely suppressed the spontaneous firing, as shown in Figure $3 B$. However, even very large hyperpolarizations (20-30 $\mathrm{mV}$ below the baseline) could not alter the rate of occurrence of spontaneous depolarizing potentials. These potentials did sometimes become slightly larger during the passage of hyperpolarizing currents, however (Fig. $3 B$ ). Passage of large depo- 

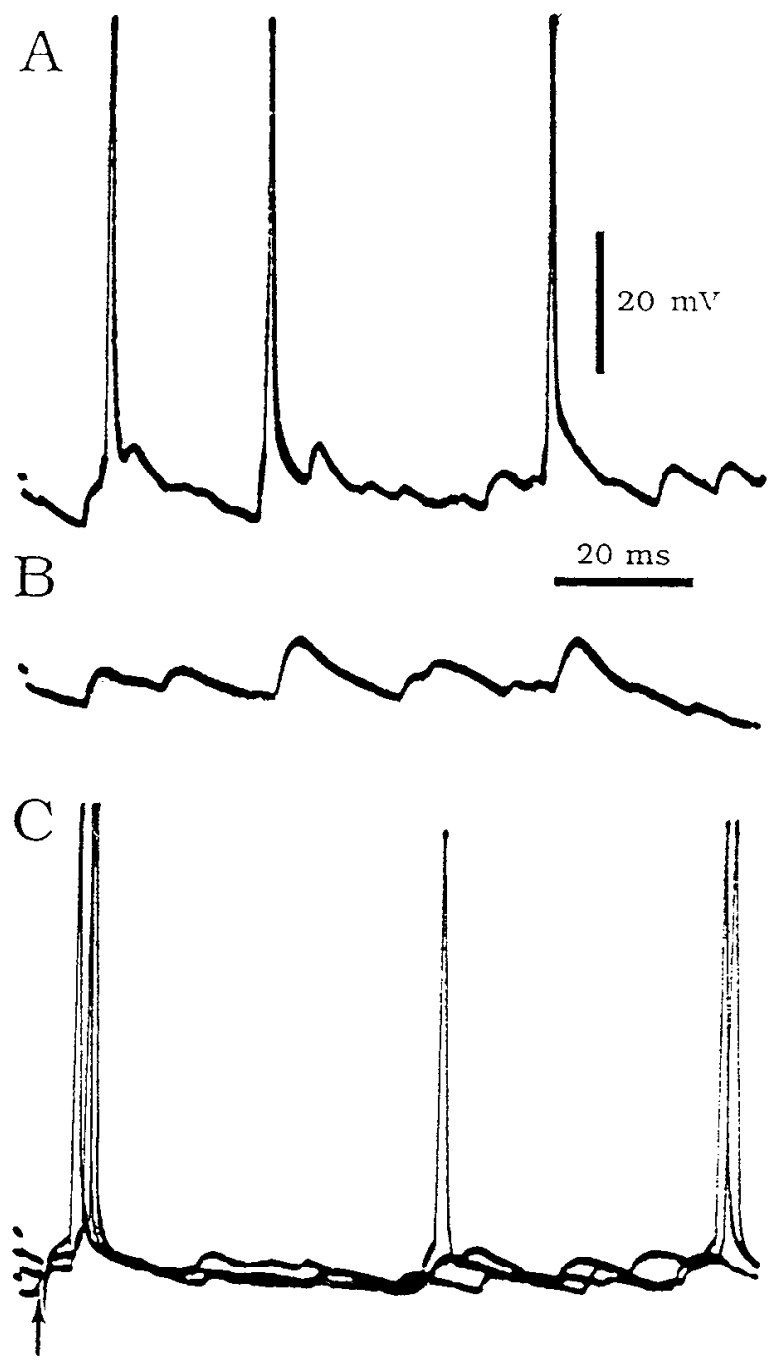

Figure 3. Evidence that the spontaneous depolarizing potentials are EPSPs. $A$, Spontaneous depolarizing potentials and action potentials in the absence of stimulation in a giant aspiny neuron. $B$, The same neuron during application of $0.5 \mathrm{nA}$ hyperpolarizing current. Action potentials are completely suppressed. The incidence of depolarizing potentials is unchanged. No amount of hyperpolarization could alter the occurrence of spontaneous depolarizing potentials in aspiny cells. Often, the amplitudes of the potentials were increased by hyperpolarization of the neuron, as they appear to be in $B$. $C$, Dependence of the depolarizing potentials on extrinsic afferent activity. Stimulation of the cerebral peduncle (at arrow) evokes an EPSP in the same neuron shown in $A$ and $B$, followed by a $50-150 \mathrm{msec}$ period of reduced incidence of spontaneous depolarizations and a corresponding reduced incidence of spontaneous action potentials. This period corresponds to the inhibitory period known to occur in corticostriatal and thalamostriatal neurons after stimulation of this kind.

larizing currents, which led to rhythmic firing in aspiny neurons (see below) did not reverse the depolarizing potentials observed in intact animals.

Measurement of the rise times and half-widths of the spontaneous depolarizing potentials was performed for 3 neurons from intact animals, in which a large number of these were recorded. Rise times and half-widths measured for depolarizing potentials that appeared to be simple and isolated did not differ for the 3 cells. Rise times varied from 1.7 to $4.7 \mathrm{msec}$ (mean $=$ 2.7 ), dnd half-widths from 4.4 to $17.0 \mathrm{msec}($ mean $=9.3 \mathrm{msec}$ ). Normalization for the average time constant for these 3 neurons

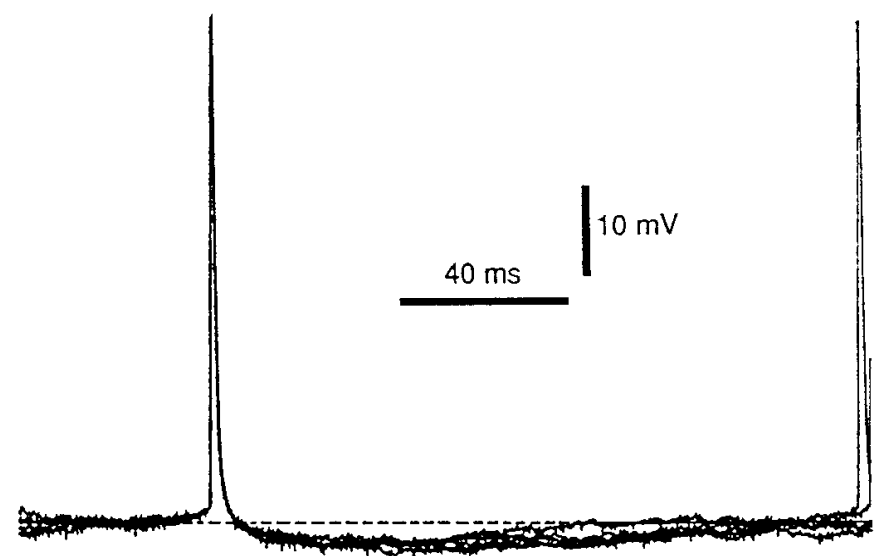

Figure 4. Spike waveform in the giant aspiny interneuron. Five spontaneously occurring action potentials are superimposed. This cell was recorded from an animal with acute decortication, accounting for the relatively low incidence of spontaneous depolarizing potentials. The neuron was firing irregularly at a mean rate of about $2 / \mathrm{sec}$, with action potentials arising from the few depolarizing potentials that remained. A membrane hyperpolarization lasting $80-120 \mathrm{msec}$ is observed to follow every action potential. Although only about $2 \mathrm{mV}$ in amplitude, this hyperpolarization was sufficient to prevent spontaneous action potentials at intervals less than $50 \mathrm{msec}$. The baseline membrane potential $(63 \mathrm{mV})$ is indicated by the dashed linc. The threshold for action potentials was $1.8 \mathrm{mV}$ above the baseline.

(19 msec) yielded a mean rise time of 0.14 and a mean halfwidth of 0.49 . Comparison with the published theoretical findings on passive neuron models and with EPSPs of known location on motor neurons and rubrospinal neurons (e.g., Rall et al., 1967; Murakami et al., 1977) suggests that if the spontaneous depolarizations were synaptic in origin, they must be located very proximally.

Several observations suggested that the spontaneous depolarizing potentials were synaptic in origin. First, the frequency of the spontaneous potentials and the rate of spontaneous firing of the neurons were much lower in the cells rccorded in animals with acute cortical lesions. Such lesions are known to remove a tonic excitatory influence on the neostriatal spiny neurons (Wilson et al., 1983b). Second, a decrease in the occurrence of spontaneous depolarizing potentials was seen to coincide with the phasic pause in neostriatal afferent synaptic activity that is known to follow stimulation of cerebral cortex, thalamus, or cerebral peduncle. This is illustrated in Figure $3 C$. The initial effect of stimulation of the cerebral peduncle is seen in that figure to be a short-lasting EPSP which triggers a single action potential. For a period of 50-100 msec thereafter, the rate of occurrence of spontaneous depolarizing potentials is decreased nearly to zero. Subsequently, these potentials, and action potentials, occur at an accelerated rate for a period of 20-50 msec. I his sequence, observed in all of the cells recorded in intact rats, corresponds in time to the sequence of long-lasting hyperpolarization and rebound depolarization that occur in spiny neostriatal neurons following this same stimulation. Those responses of the spiny neuron have been shown to be due to a period of decreased afferent synaptic activity followed by an increased synaptic bombardment from afferent fibers in the cerebral cortex and thalamus (Wilson et al., 1983b). In spiny neurons, as well as in the giant aspiny cells, these late components of the response to synchronous cortical or thalamic afferent activity are absent after acute decortication. 

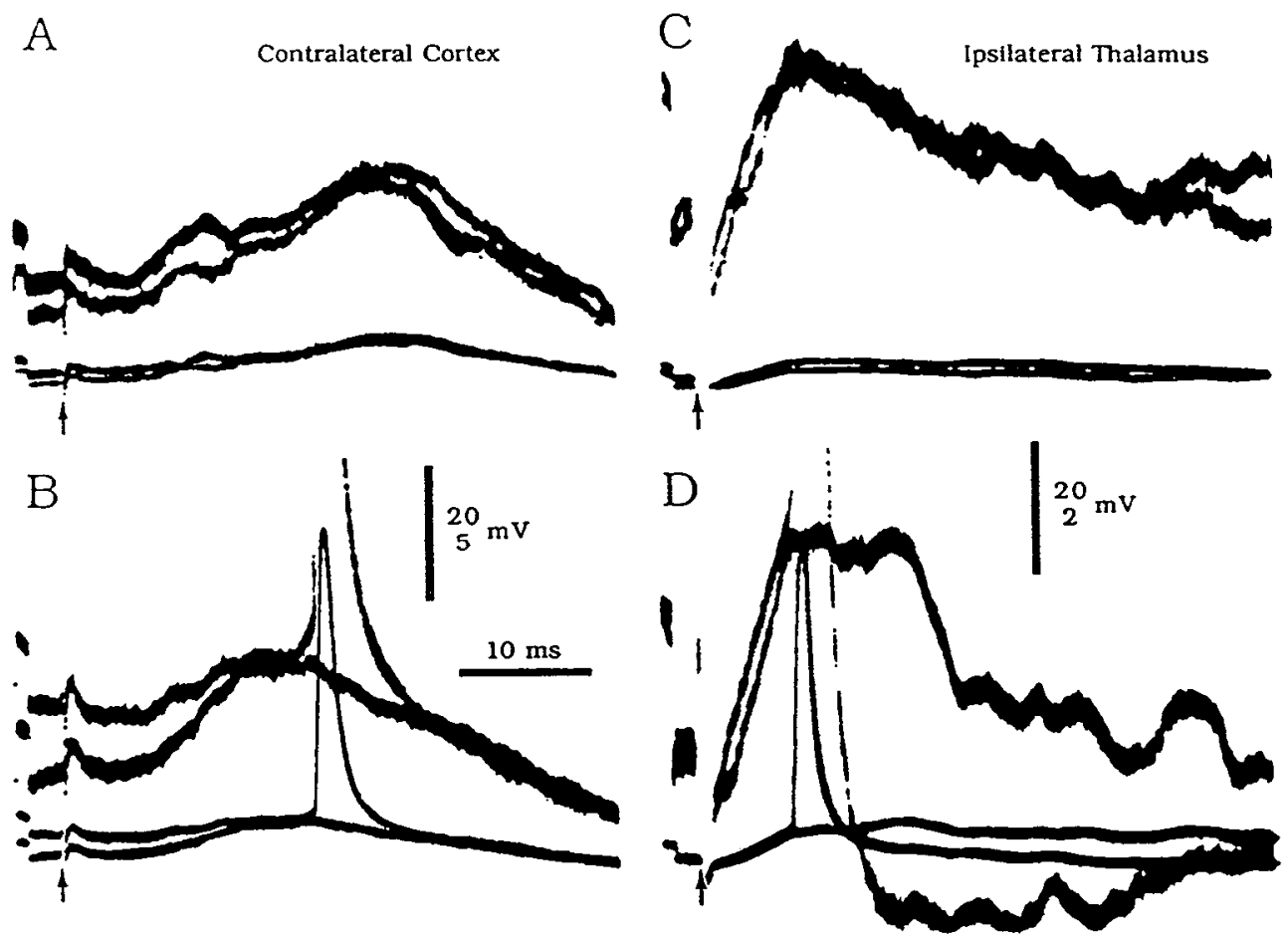

Figure 5. Convergence of afferent activity onto giant aspiny interneurons. Upper traces in all cases are AC-coupled, high-gain records. Lower traces are lower-gain DC recordings. $A$ and $B$, EPSP evoked by stimulation of the contralateral cerebral cortex. The EPSP alone is seen in $A$, as observed with justsubthreshold stimulation (at arrow). $C$ and $D$, EPSPs and action potentials evoked in the same neuron by stimulation of the thalamus. EPSP latencies in both cases match the monosynaptic EPSP latencies of the spiny neurons.

\section{Action potential shape}

The duration of action potentials of giant aspiny neurons ranged from 1.7 to $4.0 \mathrm{msec}$ (measured at the base) and were usually longer than $2.0 \mathrm{msec}$ in duration. This is substantially longer than the 1.0-2.0 msec duration of action potentials of spiny neurons recorded in the same animals. The broader action potentials were not an artifact of depolarization due to damage done by the microelectrode, because they were not substantially altered when the cells were artificially hyperpolarized and action potentials were elicited using brief depolarizing pulses. In addition to their long durations, the action potentials of the giant cells had a characteristic waveform that distinguished them from the spiny cells. The shape difference was so pronounced that it was used to correctly predict the identity of all but the first neuron in the sample. The action potential had a very fast rising phase, and a gradual decay but without any marked shoulder or inflection. These action potential features are most clearly seen in the example in Figure 4, which shows 5 superimposed spontaneous action potentials. The data shown in Figure 4 were taken from an animal with a unilateral acute decortication, so the rate of occurrence of spontaneous depolarizing potentials was very low, and the neuron was firing irregularly at a low rate $(<2 / \mathrm{sec})$. Each of the action potentials shown in Figure 4 arose from a small spontaneous depolarizing potential. The baseline membrane potential $(-63.5 \mathrm{mV})$ was only $1.8 \mathrm{mV}$ from action potential threshold. The action potentials were followed by a shallow afterhyperpolarization, about $2 \mathrm{mV}$ in amplitude at its maximum and lasting $80-100 \mathrm{msec}$. In the more active neurons observed in intact rats, the afterhyperpolarization was usually obscured by spontaneous depolarizing potentials (e.g., Fig. $3 A$ ).

\section{Synaptic potentials}

Stimulation of the ipsi- or contralateral frontal cerebral cortex, intralaminar thalamic nuclei, and cerebral peduncle evoked short-latency EPSPs in giant aspiny neurons. From our small sample of giant neurons it is not possible to make a quantitative comparison of EPSP latencies in aspiny and spiny neurons. However, the latencies of the responses of the cells in our sample were within the previously reported ranges of latencies of spiny neuron responses to the samc inputs (Wilson ct al., 1982, 1983a; Wilson, 1986). This suggests that, like the spiny cells, the giant aspiny neurons may receive monosynaptic connections from all of the major afferent pathways that converge in the neostriatum. Examples of maximal amplitude EPSPs recorded in a giant aspiny neuron are shown in Figure 5. EPSPs recorded in giant cells were much smaller in amplitude and simpler in time course than those observed in spiny neurons with the same stimulation. They were especially lacking in the complex late components that are typical of the response of neostriatal spiny neurons.

Maximal EPSP amplitudes were often less than $5 \mathrm{mV}$, and the range of stimulus intensities separating just detectable and maximal EPSPs was much smaller than seen in other neostriatal neurons. In the spiny neurons, EPSP amplitude continues to increase over a broad range of stimulus intensities, and the maximal response is often achieved only with stimuli 10-20 times threshold. In contrast, maximal stimulation of the giant cells was never more than 3-4 times the threshold for evoking the response. Stimulation near EPSP threshold often revealed a great deal of variability in the size, shape, and latency of the EPSP. This is illustrated in the example in Figure $6, A-E$. Figure $6 A$ shows the spontaneous activity of this neuron, which was recorded in an intact rat. The response to thalamic stimulation is shown at low and high gain in Figure $6 B$. The maximal EPSP had an amplitude comparable to that of the largest of the spontaneous depolarizing potentials. The latter are most clearly seen in the high-gain trace. Six consecutive responses to a stimulus near threshold for the EPSP are shown in Figure $6, C-E$. The stimulus intensity was constant and the stimulus repetition rate was $1 /$ sec. The 2 trials shown in $C$ were first in the series, 


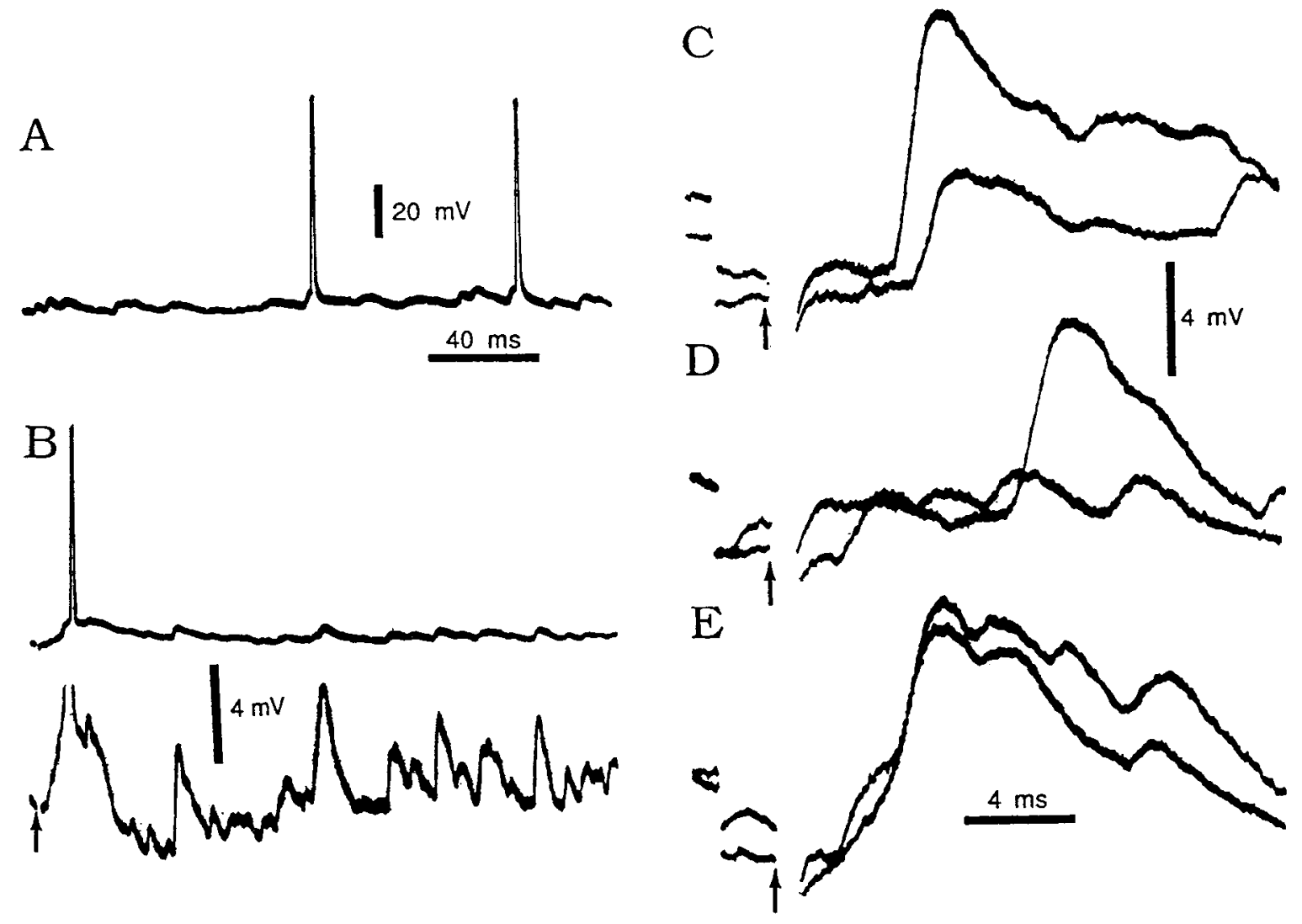

Figure 6. Spontaneous depolarizations and minimal evoked responses in a giant aspiny interneuron. $A$, Firing in the absence of a stimulus. Numerous small depolarizing potentials arc scen to occur in an irregular pattern. Most of them are subthreshold for action potentials, but some trigger spikes. $B$, The same neuron, but responding to an electrical stimulus applied to the thalamic stimulating site. A single action potential arises from an EPSP. The EPSP amplitude (approximately $2 \mathrm{mV}$ ) is slightly larger than that of the spontaneous depolarizing potentials. Upper trace is DC-coupled, at the same gain as $A$; lower trace is AC-coupled, and much higher gain to show the shape of the EPSP and the spontaneous depolarizations. $C-E$, The EPSP evoked by stimulation near threshold for the response appears to be made up of small, all-or-none depolarizing potentials similar to those observed spontaneously. Six successive sweeps, arranged in groups of 2 , are shown at high gain to illustrate the response to a stimulus applied to the cerebral peduncle. On one trial (in $D$ ), there is no response to the stimulus at all. In $C$ and $D$, the response consists of depolarizing potentials similar to the ones occurring spontaneously. They differ from each other in amplitude and latency but have similar time courses. In the last 2 traces, shown in $E$, the responses are nearly identical.

followed by the 2 shown in $D$, and finally the 2 trials shown in $E$. On one stimulus presentation shown in $D$, there was no response at all, while EPSPs evoked on the other 5 trials varied in amplitude and latency but had similar time courses. They also resembled the spontaneous depolarizing events, and an analysis of their rise times and half-widths yielded identical values to those obtained for spontaneous depolarizing potentials recorded in the same neurons (see above). It might be argued that the stimulus had no effect and that the responses shown in Figure 6, $C-E$, were simply the chance occurrence of the spontaneous depolarizing responses. However, responses to lower stimulus intensities in the same neurons show much lower frequency of occurrence of depolarizing potentials. Furthermore, increasing the stimulus intensity beyond that used in these trials produced even more reliable responses, and finally larger and longer-lasting responses such as the one shown in Figure $6 B$. Thus, the effect of increased stimulus intensity near threshold for the EPSP is hest described as increasing the probability of occurrence of depolarizing potentials indistinguishable from those that occur spontaneously. The larger EPSPs that occur with larger stimuli may result from the summation of several of the smaller depolarizing potentials occurring simultaneously.

\section{Responses to intracellularly applied current pulses}

Input resistances were determined using hyperpolarizing pulses because of the low firing threshold of the aspiny neurons, which caused the cells to fire action potentials in response to even small depolarizations. Input resistance measurements were available in 6 cells and ranged from 60 to $105 \mathrm{M} \Omega$. This is much greater than observed in spiny neurons in the same animals, which ranged from approximatcly 20 to $45 \mathrm{M} \Omega$. In addition, the aspiny cells showed much more linear steady-state responses to hyperpolarizing current than is typical for neostriatal spiny neurons.

Analysis of the repetitive firing of giant aspiny neurons in response to current pulses and of their transient responses to intracellular current pulses was completed in 4 cells, 2 of which were recorded in animals with acute decortication. The analysis was much easier in the cells recorded in decorticate animals because responses were not confounded by a high rate of spontaneous firing, but the results were essentially identical in the 4 cells. An example showing the repetitive firing of one of the cells is shown in Figure 7. In Figure $7, A-C$, firing in response to stimulus pulses ranging from 0.1 to $1.0 \mathrm{nA}$ are shown. Spike 


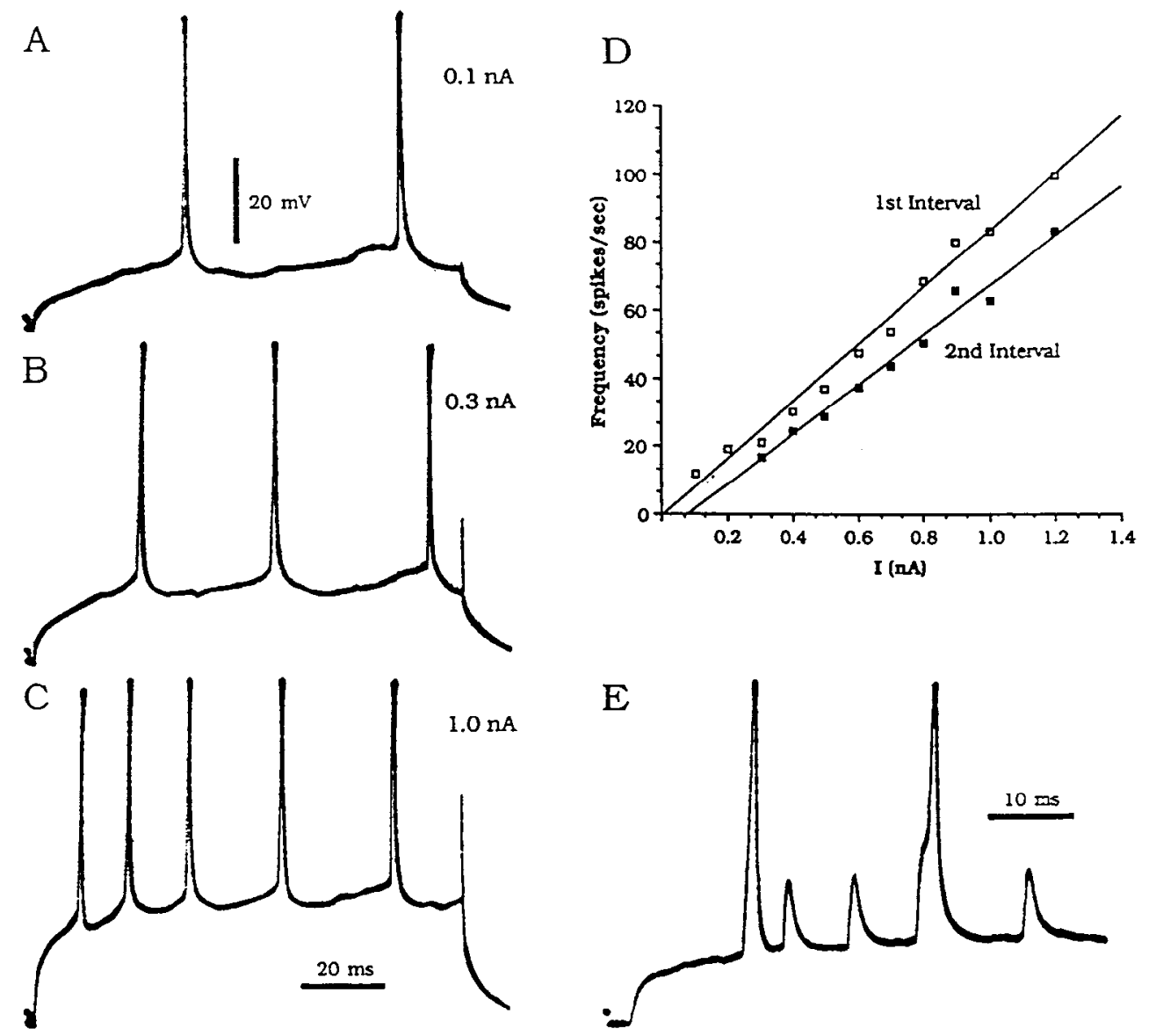

Figure 7. Repetitive firing characteristics of a giant aspiny interneuron. $A$ $C$, Responses of a neuron to constantcurrent pulses of 3 different amplitudes. $D$, Frequency-intensity relation for the full series of pulses, some of which are shown in $A-C$. Frequencies calculated from the first (open squares) and second (filled squares) intervals are plotted to show the spike frequency adaptation, which is present at all firing rates. $E$, Response of the same neuron to a 0.3 $\mathrm{nA}$ depolarizing current pulse after the cell was injected with HRP by passage of positive current pulses for $10 \mathrm{~min}$. The spike aftcrhypcrpolarization is gone, and small action potentials are intercalated between the full spikes (the latter of which continue to fire at about the normal rate). In this condition, spike repolarization was also slowed, and the membrane potential response to the onset of the pulse was more rapid. The amplitude of the action potential and the input resistance of the cell were not very different from the response observed before intracellular injection.

afterhyperpolarization was much more pronounced when superimposed upon the depolarizing current pulses but had a duration similar to that seen with spontaneous action potentials (cf. Fig. 4), and rhythmic firing began at interspike intervals of 80-100 msec, corresponding to the duration of the spike afterhyperpolarization (Fig. $7 A$ ). Spike frequency, measured as the inverse of the first interspike interval, increased linearly over the range that could be examined, as shown in Figure $7 D$. Unlike neostriatal spiny neurons, which show very little spike frequency adaptation under these circumstances, giant aspiny neurons exhibited a pronounced spike frequency adaptation, which can be seen in the comparison of frequencies calculated from the first and second interspike intervals in Figure $7 D$. These properties were shared by all 4 neurons.

In 2 of the cells examined in detail, and in several others in which repetitive firing was not analyzed, injection of HRP was followed by a change in the firing of the cells. After injection of HRP, these cells exhibited a broadening of action potentials, a reduction in spike afterhyperpolarization, an increase in the rate of spontaneous activity, and the occurrence of partial action potentials. An example of this is shown in Figure $7 E$ for the same neuron whose responses are shown in the rest of Figure 7. Partial action potentials, but still distinctly all-or-none, occurred at very short intervals, and sometimes were visible as initial components of the full action potentials (Fig. $7 E$ ). The amplitude of the full action potentials was not affected, but their duration was greatly increased, and the firing rate, as counted by the occurrence of the full spikes, was also greatly increased.
The input resistance of the neurons was not greatly altered (the current pulse in Fig. $7 E$ was the same as that in Fig. $7 \mathrm{C}$ ), but the membrane potential transient response had a much steeper onset (compare Fig. 7, $C$ and $E$ ). These effects of intracellular injection of HRP (and presumably Tris and potassium ions as well) lasted 1-10 min, after which the firing of the neurons returned to their preinjection state.

Responses to step currents were analyzed in 4 neurons using the method described by Rall (1969). As before, 2 of these neurons were from animals subjected to acute decortication. An example showing a typical result is shown in Figure 8 . The transient used for the analysis shown is in the inset at the upper right. In the lower graph, the transient is shown after normalization for size and resting membrane potential. The log of the normalized transient is plotted as a function of time. This is equivalent to plotting the $\log$ of the derivative of the transient if the measurement of the steady-state membrane potential is accurate (Rall, 1969), but it is less sensitive to noise. This method has been used previously with striatal neurons by Kita et al. (1984). The membrane time constant, estimated from the slope of the late linear portion of the transient in Figure 8, was 18.6 msec. A second exponential process, with a time constant of $2.6 \mathrm{msec}$ could also be extracted from this transient. Both offset and onset transients were analyzed for each neuron, and at least 4 transients from each neuron were analyzed. Onset and offset transients for hyperpolarizing current pulses less than $0.5 \mathrm{nA}$ superimposed exactly for all cells. With current pulses in this range, the input resistances and time constants of the neurons 
Figure 8. Analysis of time constants of a giant aspiny neuron from the transient response to a hyperpolarizing current step. The transient is shown in the inset at the upper right. The upper trace is the membrane potential transient, and the lower trace shows the current step. The amplitude of the current was 0.2 $\mathrm{nA}$. The input resistance of this neuron was $97 \mathrm{M} \Omega$ as measured from this pulse (at $100 \mathrm{msec}$ after onset). The same transient is plotted below as the log of the proportional deviation of the membrane potential from its final value (again measured $100 \mathrm{msec}$ after onset). The membrane time constant was determined as the slope of the regression line through the late linear component of the transient (line labeled $\tau_{0}=18.6$ $\mathrm{msec}$ ). Deviations from this line were again plotted on the same coordinates, and a regression line calculated for the later linear portion of this data set (line labeled $\tau_{1}=2.6 \mathrm{msec}$ ). This was taken to be the first equalizing time constant. Higher-order exponential components of the response were not extracted.

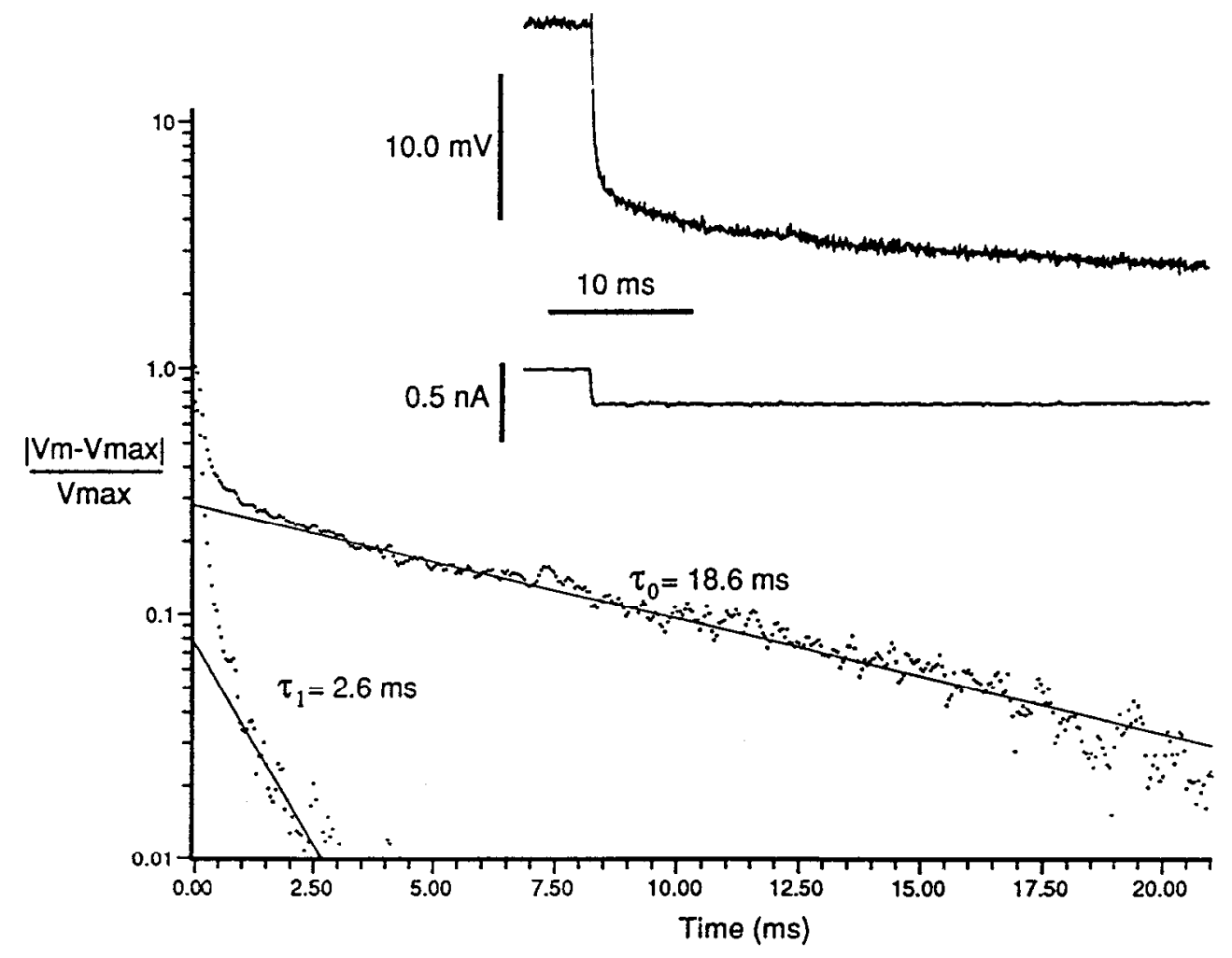

did not change systematically with the size of the current pulse used to measure them. For example, in the neuron shown in Figure 8, the input resistance obtained with the transient shown was $97 \mathrm{M} \Omega$, and the time constant $18.6 \mathrm{msec}$. Analysis of the response to a $0.4 \mathrm{nA}$ pulse yiclded an input resistance of 100 $\mathrm{M} \Omega$ and a time constant of 21.5 , while the response to a $0.3 \mathrm{nA}$ pulse gave an input resistance of $91 \mathrm{M} \Omega$ and a membrane time constant of $17.8 \mathrm{msec}$. The 2 exponential components extracted from the transient shown in Figure 8, and others from this neuron, satisfied the constraint described by Kawato (1984), that $E_{1} / E_{0}<2^{*}\left(\tau_{1} / \tau_{0}\right)$, where $E_{1}$ and $E_{0}$ are the coefficients, and $\tau_{1}$ and $\tau_{0}$ are the time constants of the first 2 exponential processes. Transients satisfying this constraint may be analyzed using the methods developed for equivalent cylinder models of neurons to yield estimates of the dendritic length and dendritic dominance ratio. That analysis was performed as described by Kawato (1984) for the neuron in Figure 8, and it yielded an estimate of 1.2 length constants for the dendritic length and a dendritic dominance of 11.0 .

Time constants for the other neurons analyzed in this way were 25.2, 22.8, and 11.0. None of these cells met Kawato's criterion for analysis using an equivalent cylinder model. They yielded electrotonic lengths of 1.5, 1.4, and 1.1 length constants when calculated using the simpler procedure of Rall (1969), which is valid when the dendritic dominance is large. The transients could also be fit using a model with nonuniform membrane resistance. When analyzed using Kawato's somatic shunt model, the results from these cells again indicated dendritic lengths between 1.0 and 1.5 , but the estimate for dendritic dominance was lower, being less than 2 for one cell and between 2 and 4 for the others, with somatic resistivity between 3 and 4 times less than that of the dendrites (the wide tolerances are due to the sensitivity of the measured coefficients to noise and of the resulting variation among traces taken from the same cell). The somatic shunt conductance measured in this way was greater for the cells recorded in the intact animal. The cell fitting the uniform resistivity model and shown in Figure 8 was recorded in an acutely decorticate rat.

\section{Discussion}

\section{Identity of the cells}

In some especially simple views of neostriatal architecture, the cells are divided into only 2 categories, large cells and small cells. The variety of morphological features seen in Golgi-stained neostriatal cells has always argued against this simple scheme, but recent advances in the identification of cell classes in the neostriatum using immunocytochemical techniques have made it untenable. Although most medium-sized neurons are spiny projection neurons, medium-sized interneurons of a variety of types can be demonstrated by staining designed to localize somatostatin, GABA, neuropeptide $Y$, and a variety of other markers (e.g., see review by Gerfen, 1988). Likewise, there are several different kinds of large neurons. Probably the largest cells are those that contain choline acetyltransferase (e.g., Bolam et al., 1984; Phelps et al., 1985). However, there are other cells with large somata which are not positive for ChAT. One class of these cells is a projection neuron with morphological features similar to globus pallidus cells (Bolam et al., 1981). Another consists of the larger instances of the class of GABAergic interneuron that contains the calcium-buffering protein parvalbumin (Cowan et al., 1987).

The cells described in this study had somata that placed them at the extreme upper end of the somatic size spectrum in the rat neostriatum. Their somatic sizes and somatodendritic morphology matched that of neostriatal cholinergic neurons. In ad- 
dition, we have examined the cytological fcaturcs, distribution of synapses on the cell surfaces, and the morphology and synaptic targets of these cells, and observed that they show exactly the features reported previously for ChAT-containing neurons in the neostriatum (these results will be published in detail elsewhere). While these observations do not rule out the possibility of an unknown interneuron type with morphological features identical to the cholinergic interneuron, it is most likely that the cells reported here are neostriatal cholinergic neurons.

The spontaneous firing patterns of these neurons are almost identical to those of the tonically firing neurons in the neostriatum of awake monkeys as described by Kimura et al. (1984). Since tonically firing neurons represent a small proportion of all cells recorded in behaving animals, it has been speculated that these cells correspond to cholinergic interneurons (Kimura et al., 1984). While the diversity of neostriatal aspiny neurons may cast suspicion on such a spcculation, the results presented here support the view that large, almost certainly cholinergic, interneurons do fire tonically in the fashion described in extracellular recording studies. On the other hand, the proportion of tonically active neurons recorded in behaving animals is much larger than the proportion of cholinergic interneurons in the same animal ( $41 \%$ of the sample reported by Kimura, 1986). It is therefore likely that there are also noncholinergic tonically firing neurons yet to be demonstrated.

\section{Afferent input}

In behaving monkeys, tonically firing neostriatal neurons respond differently from the majority of neurons during performance of a learned motor task. Whereas most neurons fire in a burst of action potentials time-locked to the onset of the movement, the tonically firing neurons are activated (or inhibited) by presentation of the sensory stimulus that acts as a signal to the animal that he should begin the movement (Kimura, 1986). It seems likely, therefore, that these neurons receive different afferent information than the spiny cells, as well as being different in the way that they process the information they do receive. The intracellular recording experiments here do not provide a direct demonstration of any such difference. The latency of EPSPs evoked in giant interneurons by stimulation of cerebral cortex, cerebral peduncle, or thalamus were well within the range observed in recordings of spiny neurons made in the same animals (Wilson et al., 1982, 1983a, b; Wilson, 1986).

There are reasons to suspect some differences, however. The large size of the dendritic field of the giant aspiny interneuron brings the cell into potential contact with many more cortical axons than possible for spiny neurons. Despite this, the giant aspiny cells appear to receive input from fewer cortical axons than the spiny cells. There are several observations that point to this conclusion. Firstly, responses to cortical inputs were neither as large nor as reliably observed in giant interneurons. In two of the neurons reported here, there was no response to the cortical stimulation sites available in the experiment. In these same animals, all spiny neurons, including some recorded very near the giant cells, showed large and reliable responses to the same cortical stimuli. In both of these aspiny cells, an EPSP was observed in response to stimulation of the cerebral peduncle, suggesting that they received cortical inputs from some region of the cortex other than those areas stimulated. None of the EPSPs to afferent stimulation were as large as those routinely observed in spiny neuron. Bishop et al. (1982), reporting on one large interneuron, interpreted the lack of a response in that cell as suggesting that cortical inputs did not impinge on giant cells at all.

A second reason for suspecting that cortical inputs to giant cells involve fewer axons than those going to projection neurons comes from the appearance of the EPSPs themselves. Even EPSPs near maximal for these responses appear to be made up of discrete elemental responses that are identical in shape and size to spontaneously occurring synaptic potentials. These elemental units have the appearance of unitary synaptic potentials. The maximal evoked response is made up of only a few (1-10) of these smaller EPSPs. The large size and rapid time course of these responses suggest that they may arise from synapses located on the soma or the most proximal portions of the dendrites. If they are unitary synaptic potentials from proximal synapses, then the maximal cortical input in the giant interneurons arises from activation of only a few cortical axons, many fewer than could be accounted for by a possible difference in the receptive surface areas of spiny and aspiny neurons. If, as reasoned above, the cortical input to giant aspiny neurons arises from only a very small subset of corticostriatal axons, it is very likely that these axons represent a special group or cortical efferents with special properties, and which could account for the unique responses of the giant interneurons to sensory cues during behavioral experiments.

\section{Integrative properties}

The differences in evoked afferent EPSPs in the spiny neurons and giant aspiny interneurons also suggest essential differences in the way these neurons process synaptic input. Spiny neurons receive many convergent excitatory synaptic connections from widespread regions of the cerebral cortex, thalamus and other areas of the brain. The effects of individual synaptic events are usually not strong enough to allow their identification in ordinary intracellular recording experiments. Correlated activity in many inputs is very important in determining the firing of spiny neurons, as indicated by their late responses to afferent stimulation (Wilson et al., 1983b). These later response components are much less visible in the responses of giant aspiny cells.

Spiny neurons have resting potentials far from the action potential threshold, at lcast in immobile animals, and action potentials in these cells arise from large synaptic potentials that are due to combined action of many synaptic potentials (Wilson and Groves, 1981). In contrast, some individual excitatory synaptic events are much more powerful in giant aspiny neurons, and appear as spontaneous, easily recorded depolarizations. The aspiny neurons' membrane potentials are normally within a few millivolts of the spike threshold, so that these unitary synaptic potentials may individually trigger action potentials. Thus the giant aspiny neuron is very sensitive to a few cortical and thalamic axons, while the spiny neurons around it are sampling from a much larger population of axons and are relatively insensitive to activity in each one.

The sensitivity of the aspiny neurons to individual synaptic inputs suggests that these inputs are placed in an electrotonically proximal region of the dendritic tree. Measurement of the electrotonic length of the dendrites using current pulses indicated that these cells do not have an especially compact dendritic field. This measurement is made using the somatic to dendritic transfer of charge, and so is not very reliable for the prediction of the effective electrotonic distance for synaptic potentials transferring charge in the opposite direction (e.g., Wilson, 1988). However, asymmetry of electrotonic distance for signals prop- 
agating in opposite directions in the dendritic field is usually associated with voltage sensitive conductances in the dendrites. There is no indication that such conductances are contributing greatly to the propagation of synaptic potentials in the giant aspiny neurons. Large depolarizations or hyperpolarizations of the somatic membrane by applied current affected the sizes of the spontaneous and evoked depolarizing potentials in the way expected for simple synaptic potentials. The somata of the giant aspiny neuron exhibited little nonlinear behavior, at least in comparison to the spiny neuron. This interpretation is also supported by our analysis of the shapes of the spontaneous depolarizations, which suggest that they have a very proximal, perhaps somatic, placement on the neurons. Thus it is most likely that the spontaneous depolarizations, and the excitatory synaptic potentials observed in these neurons, are produced by synaptic contacts on the somata or proximal parts of the dendritic trees of the cells. These findings are consistent with the observation that asymmetrical afferent-type synaptic contacts are present on proximal parts of cholinergic interneurons (e.g., Phelps et al., 1985).

The long time constant, high input resistance, and the placement of the action potential threshold so close to the baseline membrane potential of the giant aspiny neurons also make them sensitive to excitatory synaptic inputs with proximal locations.

\section{Repetitive firing}

Although tonically active, giant aspiny neurons in the neostriatum have not been observed to fire rhythmically under ordinary conditions. The reason for this is apparent upon examination of the membrane potential trajectory of the neurons during their spontaneous activity. The firing of the neurons is patterned by the occurrence of spontaneous depolarizing potentials, rather than by pacemaker potentials whosc cyclic gencration is reset by the action potential generation. For the cells to fire rhythmically, either the spontaneous potentials would have to become rhythmic, or the cell would have to be depolarized so that its firing was determined by spike afterhyperpolarization (as happens during rhythmic firing in response to long artificially applied current pulses). Still, spike afterhyperpolarization apparently acts to limit the firing rate that can be maintained by the cells in response to their normal inputs. In the cells studied here, the majority of spontaneous depolarizing potentials were subthreshold for spike generation, with only a small proportion, usually a combination of more than one smaller depolarization attaining threshold for action potential generation. During the 80-100 msec period of afterhyperpolarization that follows a spike, spontaneous depolarizations are even less likely to fire action potentials. Thus under most conditions, the spike afterhypcrpolarization is sufficient to limit firing of the aspiny neurons to rates of $16 / \mathrm{sec}$ or less. Maintained firing at these rates will engage another limiting factor, the spike frequency adaptation that was consistently seen in these cells during repetitive firing.

The mechanisms of these two firing rate limiting factors may be related, since they were both reversibly deactivated by the prolonged depolarizing current pulses used for injection of IIRP. Two additional effects of these currents were (1) an increase in the duration of action potentials, and (2) an increase in linearity of the transient response to depolarizing current pulses resulting in an increase in the apparent time constant of the initial part of the charging curve. All of these effects disappeared a few minutes after injection of HRP was discontinued. Because of their short duration the effects of intracellular injection are morc likely to be due to potassium loading rather than loading with HRP. It is possible that the potassium concentration was restored in a few minutes, but the HRP remained in the cytoplasm of the cells for several hours, as indicated by intracellular staining.

\section{Effects on neostriatal output}

The effect exerted by the giant aspiny interneurons on the spiny projection cells of the neostriatum is unknown. Studies of the effects of acetylcholine on spiny neurons have suggested a possible fast nicotinic excitation, and also a muscarinic response that results in a decreased sensitivity to excitatory synaptic activation (e.g., Dodt and Misgeld, 1986; Akaike et al., 1988). In addition, however, there are reports of modulatory effects of muscarinic agonists, both on the duration of calcium-dependent platcau potentials and, at higher doses, on input resistance (e.g., Misgeld et al., 1986). Because the giant cells are tonically active, they are in a position to maintain a relatively constant level of cholinergic input to the spiny cells, and so are in a position to act as continuous modulators of the excitability of the output cells. Their greater sensitivity to the few corticostriatal inputs they receive may underly their responses to sensory stimuli associated with the timing of voluntary movements (Kimura, 1986), and results in changes in their firing prior to those of the spiny neurons during the initiation of movement. This may serve to prepare the projection neurons for the afferent input barrage that is more directly related to the movement itself, and so create a sensory-motor preparatory set within the circuitry of the neostriatum.

\section{References}

Akaike, A., M. Sasa, and S. Takaori (1988) Muscarinic inhibition as a dominant role in cholinergic regulation of transmission in the caudate nucleus. J. Pharm. Exp. 246: 1129-1136.

Bishop, G. A., H. T. Chang, and S. T. Kitai (1982) Morphological and physiological properties of neostriatal neurons: An intracellular horseradish peroxidase study in the rat. Neuroscience 7: 179-191.

Bolam, J. P., J. F. Powell, S. Totterdell, and A. D. Smith (1981) A second type of striatonigral neuron: A comparison between retrogradely labelled and Golgi-stained neurons at the light and electron microscopic levels. Neuroscience 6: 2141-2157.

Bolam, J. P., B. H. Wainer, and A. D. Smith (1984) Characterization of cholinergic neurons in the rat neostriatum. A combination of choline acetyltransferase immunocytochemistry, Golgi-impregnation and electron microscopy. Neuroscience 12: 711-718.

Chang, H. T., C. J. Wilson, and S. T. Kitai (1982) A Golgi study of rat neostriatal neurons: Light microscopic analysis. J. Comp. Neurol. 208: $107-126$.

Cowan, R. L., C. J. Wilson, and P. C. Emson (1987) Parvalbumin is present in GABA containing interneurons of the rat neostriatum. Soc. Neurosci. Abstr. 13: 1573.

DeLong, M. R. (1973) Putamen: Activity of single units during slow and rapid arm movements. Science 179: 1240-1242.

DiFiglia, M. (1987) Synaptic organization of cholinergic neurons in the monkey neostriatum. J. Comp. Neurol. 225: 245-258.

DiFiglia, M., P. Pasik, and T. Pasik (1976) A Golgi study of neuronal types in the neostriatum of monkeys. Brain Res. 114: 245-256.

Dodt, H. U., and U. Misgeld (1986) Muscarinic slow excitation and muscarinic inhibition of synaptic transmission in the rat neostriatum. J. Physiol. (Lond.) 380: 593-608.

Fuller, D. R. G., C. D. Hull, and N. A. Buchwald (1975) Intracellular responses of caudate output neurons to orthodromic stimulation. Brain Res. 96: 337-341.

Galarraga, E., J. Bargas, A. Sierra, and J. Aceves (1989) The role of calcium in the repetitive firing of neostriatal neurons. Exp. Brain Res. (in press). 
Gerfen, C. R. (1988) Synaptic organization of the striatum. J. Elect. Microsc. Tech. 10: 265-281.

Graveland, G. A., and M. DiFiglia (1985) The frequency and distribution of medium-sized neurons with indented nuclei in the primate and rodent neostriatum. Brain Res. 327: 308-311.

Grofova, I., J. M. Deniau, and S. T. Kitai (1982) Morphology of the substantia nigra pars reticulata projection neurons intracellularly labeled with HRP. J. Comp. Neurol. 208: 352-368.

Groves, P. M. (1983) A theory of the functional organization of the neostriatum and the neostriatal control of voluntary movement. Brain Res. Rev. 5: 109-132.

Horikawa, H., and W. E. Armstrong (1988) A versatile means of intracellular labeling: Injection of biocytin and its detection with avidin conjugates. J. Neurosci. Methods 25: 1-11.

Kawato, M. (1984) Cable properties of a neuron model with nonuniform membrane resistivity. J. Theor. Biol. 111: 149-169.

Kemp, J. M., and T. P. S. Powell (1971) The structure of the caudate nucleus of the cat: Light and electron microscopy. Phil. Trans. R. Soc. London [Biol.] 262: 383-401.

Kimura, M. (1986) The role of primate putamen neurons in the association of sensory stimuli with movement. Neurosci. Res. 3: 436443.

Kimura, M., J. Raijkowski, and E. Evarts (1984) Tonically discharging putamen neurons exhibit set-dependent responses. Proc. Natl. Acad. Sci. USA 81: 4998-5001.

Kita, T., H. Kita, and S. T. Kitai (1984) Passive electrical membrane properties of rat neostriatal neurons in in vitro slice preparation. Brain Res. 300: 129-139.

Liles, S. L. (1974) Single-unit responses of caudate neurons to stimulation of frontal cortex, substantia nigra and entopeduncular nucleus in cats. J. Neurophysiol. 37: 254-265.

Misgcld, U., P. Calabrcsi, and H. U. Dodt (1986) Muscarinic modulation of calcium-dependent plateau potentials in rat neostriatal neurons. Pflügers Arch. 407: 482-487.

Murakami, F., N. Tsukahara, and Y. Fujito (1977) Analysis of unitary EPSP mediated by the newly formed cortico-rubral synapses after lesion of the nucleus interpositus of the cerebellum. Exp. Brain Res. 30: 233-243.

Phelps, P. E., C. R. Houser, and J. E. Vaughn (1985) Immunocytochemical localization of choline acetyltransferase within the rat neostriatum: A correlated light and electron microscopic study of cholinergic neurons and synapses. J. Comp. Neurol. 238: 286-307.

Pickel, V. M., K. K. Sumal, S. C. Beckley, R. J. Miller, and D. J. Reis (1980) Immunocytochemical localization of enkephalin in the neostriatum of rat brain: A light and electron microscopic study. J. Comp. Neurol. 189: 721-740.

Preston, R. J., G. A. Bishop, and S. T. Kitai (1980) Medium spiny neurons projection from the rat striatum: An intracellular horseradish peroxidase study. Brain Res. 185: 253-263.

Rall, W. (1969) Time constants and electrotonic length of membrane cylinders and neurons. Biophys. J. 9: 1483-1508.

Rall, W., R. E. Burke, T. G. Smith, P. G. Nelson, and K. Frank (1967) Dendritic location of synapses and possible mechanisms for the monosynaptic EPSP in motoneurons. J. Neurophysiol. 30: 1169-1 193.

Wilson, C. J. (1986) Postsynaptic potentials evoked in spiny neostriatal projection neurons by stimulation of ipsilateral and contralateral cortex. Brain Res. 367: 201-213.

Wilson, C. J. (1988) Cellular mechanisms controlling the strength of synapses. J. Electr. Microsc. Tech. 10: 293-313.

Wilson, C. J., and P. M. Groves (1981) Spontaneous firing patterns of identified spiny neurons in the rat neostriatum. Brain Res. 220: $67-80$.

Wilson, C. J., H. T. Chang, and S. T. Kitai (1982) Origins of postsynaptic potentials evoked in identified rat neostriatal neurons by stimulation in substantia nigra. Exp. Brain Rcs. 45: 157-167.

Wilson, C. J., H. T. Chang, and S. T. Kitai (1983a) Origins of postsynaptic potentials evoked in spiny neostriatal projection neurons by thalamic stimulation in the rat. Exp. Brain Res. 51: 217-226.

Wilson, C. J., H. T. Chang, and S. T. Kitai (1983b) Disfacilitation and long-lasting inhibition of neostriatal neurons in the rat. Exp. Brain Res. 51:217-226. 